\title{
Learning from Errors: Error-Related Neural Activity Predicts Improvements in Future Inhibitory Control Performance
}

\author{
Robert Hester, ${ }^{1,2}$ Janelle Madeley, ${ }^{2}$ Kevin Murphy, ${ }^{3}$ and Jason B. Mattingley ${ }^{2}$ \\ ${ }^{1}$ Department of Psychology, School of Behavioural Science, University of Melbourne, Melbourne, Victoria 3010, Australia, ${ }^{2}$ Cognitive Neuroscience \\ Laboratory, Queensland Brain Institute and School of Psychology, University of Queensland, St Lucia, Queensland 4072, Australia, and ${ }^{3}$ Cardiff University \\ Brain Research Imaging Centre, School of Psychology, Cardiff University, CF10 3AT Cardiff, United Kingdom
}

Failure to adapt performance following an error is a debilitating symptom of many neurological and psychiatric conditions. Healthy individuals readily adapt their behavior in response to an error, an ability thought to be subserved by the posterior medial frontal cortex (pMFC). However, it remains unclear how humans adaptively alter cognitive control behavior when they reencounter situations that were previously failed minutes or days ago. Using functional magnetic resonance imaging, we examined neural activity during a Go/No-go response inhibition task that provided the opportunity for participants to learn from their errors. When they failed to inhibit their response, they were shown the same target stimulus during the next No-go trial, which itself could occur up to 20 trials after its initial presentation. Activity within the pMFC was significantly greater for initial errors that were subsequently corrected than for errors that were repeated later in the display sequence. Moreover, pMFC activity during errors predicted future responses despite a sizeable interval (on average 12 trials) between an error and the next No-go stimulus. Our results indicate that changes in cognitive control performance can be predicted using error-related activity. The increased likelihood of adaptive changes occurring during periods of recent success is consistent with models of error-related activity that argue for the influence of outcome expectancy (Holroyd and Coles, 2002; Brown and Braver, 2005). The findings may also help to explain the diminished error-related neural activity in such clinical conditions as schizophrenia, as well as the propensity for perseverative behavior in these clinical groups.

\section{Introduction}

The question of how the human brain detects errors and adapts subsequent behavior, often in the absence of overt reinforcement or feedback, has been widely studied within cognitive neuroscience for more than two decades (Falkenstein et al., 1991; Ridderinkhof et al., 2004). A range of evidence implicates the posterior medial frontal cortex (pMFC) in error processing (Ullsperger and Von Cramon, 2003; Ridderinkhof et al., 2004). The magnitude of neural activity in the pMFC has been related to adaptive posterror changes in response behavior, including improvement in response speed, which suggests increases in cognitive control (Gehring et al., 1993; Kerns et al., 2004), and generalized slowing of responding, which is argued to reflect more cautious posterror behavior (Garavan et al., 2002; Debener et al., 2005).

Work to date has focused on immediate posterror changes in cognitive control behavior, in which there is close temporal proximity between the error and behavior change. The limitation of

\footnotetext{
Received Sept. 10, 2008; revised March 1, 2009; accepted March 2, 2009.

This research was supported by Australian Research Council Grant DP0556602 (R.H.) and National Health and Medical Research Council Fellowship 519730 (R.H.). The assistance of Drs. Katie McMahon, Mark Strudwick, and Matt Meredith (Wesley Hospital) and of Dr. Mark Bellgrove, Felicity Brown, and Ashley Skilleter is gratefully acknowledged.

Correspondence should be addressed to Dr. Robert Hester, Department of Psychology, School of Behavioural Science, Redmond Barry Building, University of Melbourne, Melbourne, VIC 3010, Australia. E-mail: hesterr@unimelb.edu.au.

DOI:10.1523/JNEUROSCI.4337-08.2009

Copyright $\odot 2009$ Society for Neuroscience $\quad$ 0270-6474/09/297158-08\$15.00/0
}

this work is that it remains unclear whether the association between error-related pMFC activity and adaptive change in executive control performance is relatively crude-associated simply with the invocation of a general state of increased cognitive control-or whether it also relates to delayed, stimulus-specific alterations in posterror performance. Although one of the hallmarks of human cognitive control behavior is the ability to immediately and dramatically alter behavior following an error, humans are also capable of adaptively altering cognitive control behavior when they reencounter situations that were previously failed minutes, days, or weeks ago.

Several influential models have argued for a relationship between error-related pMFC activity and adaptive changes in performance, via dopaminergically mediated reinforcement learning mechanisms (Holroyd and Coles, 2002; Brown and Braver, 2005). These models argue that error-related neural activity is associated with delayed adaptive posterror changes to cognitive control. Research to date had identified an association between the magnitude of error-related activity and subsequent learning performance (Klein et al., 2007b; Hester et al., 2008), but has failed to show the expected relationship to cognitive control, measured indirectly via adaptive posterror slowing (Hester et al., 2007).

To examine the relationship between error-related neural activity and delayed adaptive posterror changes in a direct measure of cognitive control behavior, we administered a version of the Go/No-go response inhibition task that provided participants with the opportunity to learn from response inhibition errors 


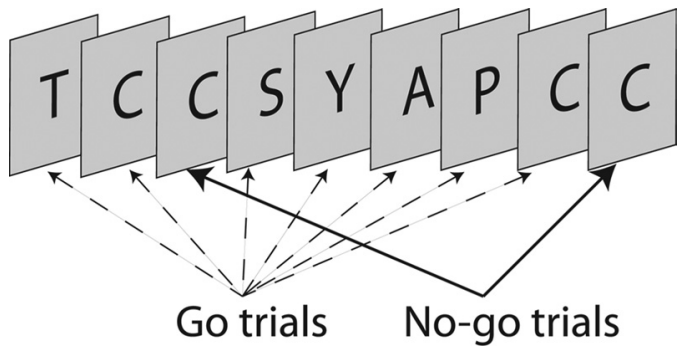

Figure 1. Sample displays from the Go/No-go task. A series of letters was displayed at a rate of 1 per second. Participants were required to respond to each of the Go-trials with a button press, and to withhold their response whenever an identical letter was presented on consecutive trials. The figure presents an example of a No-go trial (the repetition of the letter " $\mathrm{C}^{\prime \prime}$ ), followed by the presentation of the same stimulus on the next No-go trial (the second repetition of the letter " $\left.C^{\prime \prime}\right)$. This stimulus sequence only occurred when participants had unsuccessfully inhibited their response to the first No-go trial.

(Fig. 1). Participants were instructed that No-go trial performance influenced the trial sequence, such that any failure to inhibit a response during No-go trials would be followed-up to 20 trials later-by the representation of the same No-go stimulus. This manipulation provided an incentive to participants to encode the stimulus during an error, because successful encoding would allow them to predict the appearance of the next No-go trial and avoid consecutive No-go errors. We compared brain activity for correction-predicting versus error-predicting errors, categorized on the basis of subsequent performance (Fig. 2).

Commission errors were expected to elicit significant blood oxygen level-dependent (BOLD) signals from the pMFC during all No-go errors. We hypothesized that the magnitude of pMFC activity during errors would predict future performance, with significantly higher activity for correction-predicting than for error-predicting errors.

\section{Materials and Methods}

Participants. Sixteen healthy volunteers ( 10 females; mean $(M)$ age $=23$ years; range $=19-42$ years) participated in the experiment. All participants provided written informed consent, which was approved by ethics committees at The University of Queensland and Wesley Hospital (Auchenflower, Australia).

Experimental protocols. A Go/No-go response inhibition task was administered in which random letters of the alphabet were presented serially at $1 \mathrm{~Hz}$. Participants were asked to make a button response for each letter in the sequence, and to withhold their response whenever a letter was presented on consecutive trials (Fig. 1). Stimulus duration was 700 $\mathrm{ms}$ followed by a $300 \mathrm{~ms}$ blank interstimulus interval. The number of Go trials separating No-go trials ranged between 1 and $22(M=12, \mathrm{SD}=6)$, with the No-go trial gap randomly selected from this range. Only odd number No-go trial gaps were selected, to ensure that No-go trials always coincided with the beginning of an MR image acquisition. The average interval was 12.4 trials.

Participants were instructed that their performance on No-go trials would influence the task trial sequence. No-go trials occurred randomly in the trial sequence and could therefore be any letter of the alphabet. Incorrect responses to No-go trials (making the prepotent Go response) ensured the next No-go trial would present the same letter stimulus (Fig. 1). A correct response (withholding the Go response) ensured that the next No-go trial would present a different letter of the alphabet. The aim of this design was to provide an incentive to participants to encode the letter stimulus during an error, allowing them to predict the appearance of the next No-go trial and therefore to avoid making consecutive No-go trial errors. If a participant failed to inhibit on a second consecutive No-go trial, or made consecutive No-go trial errors, the No-go trial following the second error would present a different random letter of the alphabet. This ensured only two consecutive No-go trials would present the same letter of the alphabet and that a second consecutive error (repeat error) was not predictive of the next No-go trial.

Behavioral data from each participant were used to categorize the No-go trial events into successful responses (stops), correctionpredicting errors, and error-predicting errors. Errors were classified according to the response made on the subsequent presentation of the same No-go trial (Fig. 2). An incorrect No-go response that was followed by another incorrect No-go response in the subsequent No-go trial presentation was classed as an "error-predicting error." An incorrect No-go response that was followed by a stop in the following presentation was classed as a "correction-predicting error."

The second error in a pair of consecutive errors (presenting the same letter stimulus) was examined separately, with separate regressors used as before to compare errors by subsequent performance. These errors were called "repeat" errors and were further categorized into adaptive and maladaptive repeat errors. The purpose of this analysis was to compare error-related activity when the No-go stimulus did not provide predictive information [because the No-go trial following a consecutive error was always a different (random) letter of the alphabet].

Correct response inhibition during No-go trials were labeled stops and were also categorized into those presenting the same stimulus letter (stop same) or a different letter (stop different) as the previous No-go trial.

Before entering the MRI scanner, participants practiced two novel blocks of the task to ensure that they understood the task and were clear about how their performance influenced the trial sequence. During MRI data collection, eight blocks of 260 trials ( 240 Go trials, 20 No-go trials) were administered, with each block separated by a short break. All aspects of stimulus delivery and response recording were controlled by E-Prime software (version 1.1, Psychology Software Tools), running on a laptop PC (Celeron $2 \mathrm{GHz}, 128 \mathrm{MB}$ Nvidia Video Card) that was interfaced with the MR scanner during acquisition of fMRI data. Stimuli were backprojected onto a screen at the foot of the scanner bed, and a head-coilmounted mirror enabled participants to view the stimuli. Participants responded using to each stimulus using their right index finger, entering their response on a two-button MR-compatible response box (FiberOptic response pads, Current Designs).

Image acquisition. Functional MR images were acquired at the Wesley Hospital, using a whole-body 4 tesla Bruker Medspec Varian scanner with a gradient-echo echoplanar imaging (EPI) sequence. The scanner was equipped with a standard radio-frequency birdcage head coil for signal transmission and reception. Lateral head stabilizers were used to minimize head movement. EPI images were acquired using a gradientecho pulse sequence and sequential slice acquisition $(T R=2000 \mathrm{~ms}$, $\mathrm{TE}=30 \mathrm{~ms}$, flip angle $=90^{\circ}, 32$ contiguous slices of $3 \mathrm{~mm}$ thickness, $10 \%$ gap, in-plane resolution of $3.5 \times 3.5$ pixels in an FOV of $384 \mathrm{~mm}$ ). Each functional run began with two volume acquisitions that were later discarded, to allow for steady-state tissue magnetization. A total of 140 EPI volumes were collected for each functional run, and a total of eight functional runs were performed for each participant. Activation data were registered to high-resolution T1-weighted isotropic $\left(1 \mathrm{~mm}^{3}\right)$ structural MPRAGE images to localize the pattern of physiological changes associated with the task.

Data analysis. All analyses were conducted using AFNI software (http://afni.nimh.nih.gov/afni/)(Cox, 1996). Following image reconstruction, the time-series data were time shifted using Fourier interpolation to remove differences in slice acquisition times, and motion corrected using $3 \mathrm{D}$ volume registration (least-squares alignment of three translational and three rotational parameters). Activation outside the brain was also removed using edge detection techniques.

Separate hemodynamic response functions at $2 \mathrm{~s}$ temporal resolution were calculated using deconvolution techniques for correctionpredicting errors, error-predicting errors, repeat adaptive and maladaptive errors, and stop events. Response functions for all regressor events were initiated at stimulus onset, because the presentation of all eventsof-interest was timed to coincide with the beginning of the $2 \mathrm{~s}$ TR-cycle. Omission errors for Go trials and other inconsequential task events (e.g., instruction screens) were included as regressors in the model to avoid contamination of the baseline and event-related activity estimates, but 
were not subjected to further analysis. A nonlinear regression program determined the best-fitting gamma-variate function for these IRFs as previously described (Garavan et al., 1999). The area under the curve of the gamma-variate function was expressed as a percentage of the area under the baseline. The baseline in this design is an implicit one and is indicative of ongoing and remaining taskrelated Go-trial processing after the variance related to the other types of events have been removed.

The percentage area (event-related activation) map voxels were resampled at $1 \mathrm{~mm}^{3}$ resolution, then spatially normalized to standard MNI space (MNI 152 template) and spatially blurred with a $3 \mathrm{~mm}$ isotropic rms Gaussian kernel. Group activation maps for event type (correction-predicting errors, error-predicting errors, stops, repeat adaptive and maladaptive errors) were determined with one-sample $t$ tests against the null hypothesis of zero event-related activation changes (i.e., no change relative to baseline). Significant voxels passed a voxelwise statistical threshold $(t=4.31, p \leq 0.001)$ and were required to be part of a larger $142 \mu$ l cluster of contiguous significant voxels. By using a combination of probability thresholding and cluster thresholding, the aim is to maximize the power of the statistical test while holding the likelihood of false positives to a minimum. The Alphasim program (http://afni.nimh.nih.gov/pub/dist/doc/ program_help/AlphaSim.html) was used to determine the cluster threshold. The program is provided with the number of voxels in the group map, the spatial correlation of voxels (must be contiguous on three sides), and the voxelwise threshold (in this study, $p=0.001$ ). Using these values, the program conducts a series of Monte Carlo simulations (1000 iterations for our study) to determine the frequency of each conforming cluster size produced purely by chance. From this frequency distribution, the cluster size (142 $\mu$ l given our parameters) that occurs $<1 \%$ of the time by chance can be selected, to give a threshold of $p=0.01$ (corrected).

The primary comparison of interest was any difference in activation between correctionand error-predicting errors. The activation clusters from whole-brain analyses of both correction- and error-predicting errors (Table 1) were used to create an OR map for the purposes of an ROI analysis. An OR map includes the voxels of activation indicated as significant from either of the constituent maps. The mean activation for clusters in the combined map was then calculated for the purposes of an ROI analysis, deriving mean activation levels for correction- and error-predicting errors that were compared using repeated-measures $t$ tests, corrected via a modified Bonferroni procedure for multiple comparisons (Keppel, 1991).

The analyses examining repeat errors followed the same steps as above. The lower number of events in the repeat error comparison (average number of events: adaptive errors $=14.4$; maladaptive errors $=7.9)$ has the capacity to confound the spatial extent of activity in whole-brain analyses but not the estimation of peak/mean activity levels from ROIs (Murphy and Garavan, 2005). We therefore used the initial error-related
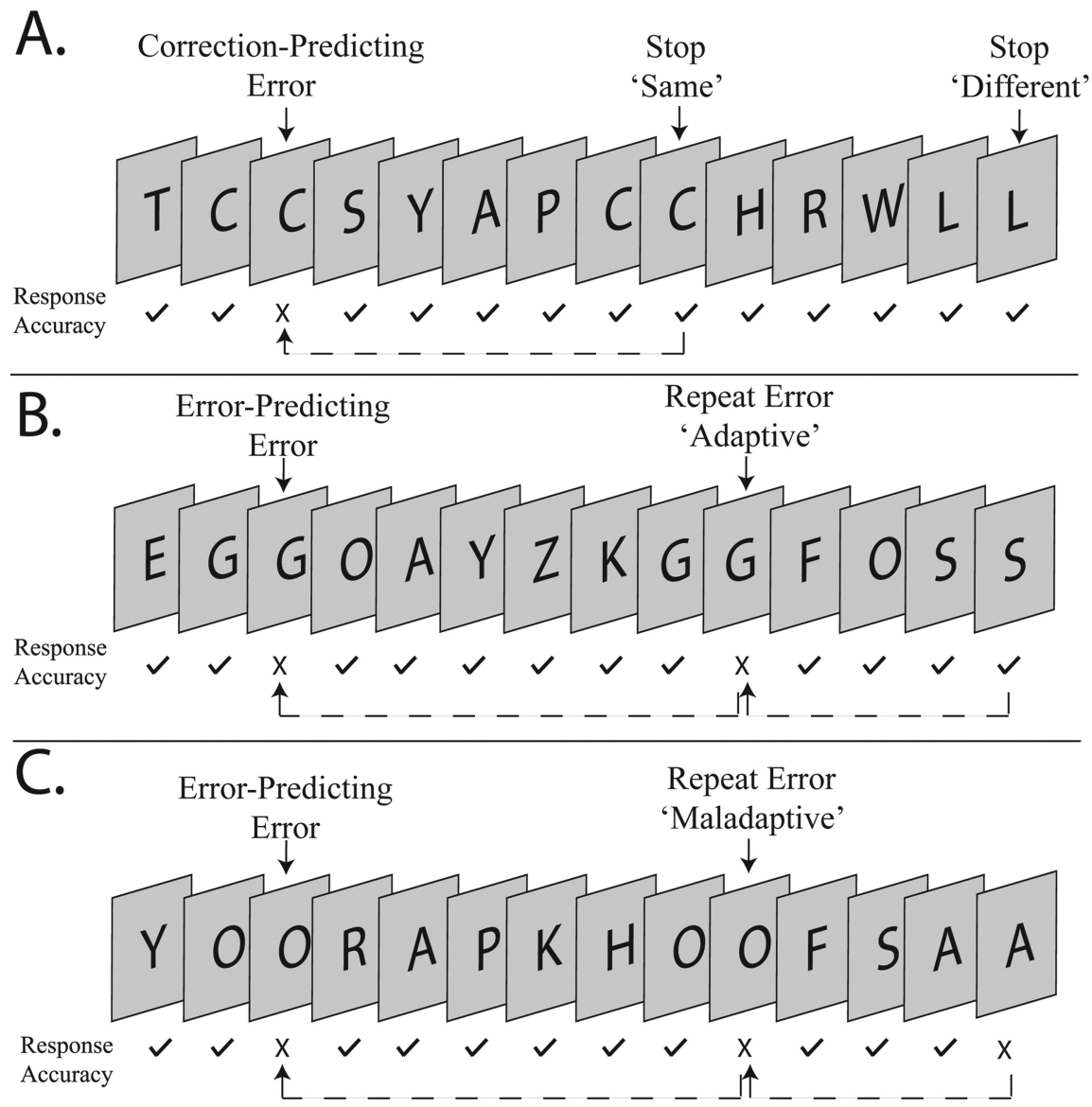

Figure 2. Classification of No-go events. No-go trials were categorized based on performance (errors, marked with a cross; and stops, marked with a tick) and by performance on the subsequent No-go trial (relationship indicated by dotted lines). Correctionpredicting errors were №-go errors that were followed by correct performance on the subsequent №-go trial $(\boldsymbol{A})$, whereas error-predicting errors were followed by another No-go error ( $\boldsymbol{B}, \boldsymbol{C}$ ). Stops (correct response inhibition) were also categorized into those presenting the same stimulus letter (stop "same") or a different letter (stop "different") as the previous No-go trial. The second consecutive failure to inhibit for the same letter stimulus was labeled as a "repeat" error, which could also be considered "adaptive" $(\boldsymbol{B})$ or "maladaptive" $(\boldsymbol{C})$ depending on performance for the subsequent No-go trial. Note that the No-go trial following a repeat error would always present a different stimulus letter.

Table 1. Regions of error-related BOLD activity differentiating correction- from error-predicting errors

\begin{tabular}{lcrrr}
\hline & & \multicolumn{3}{c}{ MNI coordinates } \\
\cline { 4 - 5 } Brain region & Volume $(\mu \mathrm{l})$ & $x$ & $y$ & $z$ \\
\hline Correction-predicting errors $>$ error-predicting errors & & 2 & 12 & 44 \\
$\quad$ R anterior cingulate & 12,932 & 40 & 14 & 5 \\
R insula & 9396 & -40 & 13 & 4 \\
L insula & 7955 & 53 & -42 & 32 \\
R inferior parietal & 6945 & -54 & -41 & 39 \\
L inferior parietal & 971 & 48 & -27 & -9 \\
R middle temporal & 636 & 49 & 39 & 12 \\
R inferior frontal & 151 & & & \\
Error-predicting errors $>$ correction-predicting errors & & 51 & -7 & -14 \\
$\quad$ R middle temporal & 190 & -4 & -54 & 19 \\
$\quad$ L posterior cingulate & 166 & & & \\
\hline
\end{tabular}

group map to perform a region-of-interest analysis comparing activity for the relevant repeat error events. The mean activation for the pMFC region was then calculated using the voxel-level percentage change estimates for each event type, deriving mean activation scores for adaptive and maladaptive repeat error types. The estimates were compared using repeated-measures $t$ tests, corrected via the modified Bonferroni procedure for multiple comparisons (Keppel, 1991). 


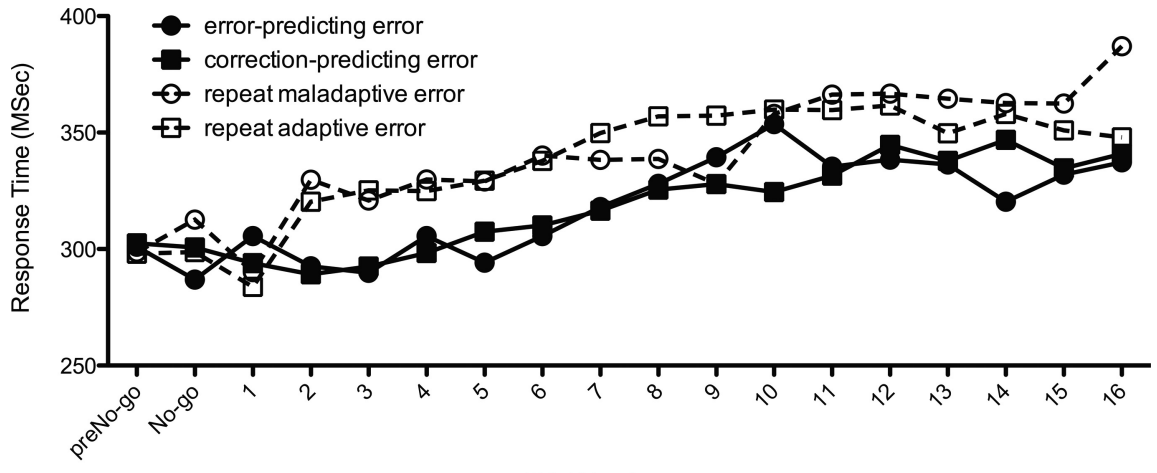

Trial Number

Figure 3. Go trial reaction times for the period following correction- and error-predicting errors. Mean reaction times are presented for the Go trial preceding a No-go error (preNo-go), the failed No-go trial (No-go), and the Go trials that followed the error. Although the number of Go trials following an error ranged from 1 to 22 , the range for the analysis was restricted to 16 trials to improve reliability. The later trials (i.e., 17-22) were excluded because some participants made too few errors to obtain a reliable estimate of response speed. Repeat errors represent the second error in a consecutive pair of No-go errors and are distinct from first-presentation errors because they were not predictive of the next No-go trial (which was a different random letter).

\section{Results}

\section{Behavioral results}

The behavioral data revealed a classic "learning from errors" repetition effect (Hebb, 1961). Response inhibition performance for No-go trials following a commission error was significantly better when the same stimulus was presented $(M=68 \%, \mathrm{SD}=19 \%)$ than when a different stimulus appeared $(M=53 \%, \mathrm{SD}=30 \%$; $\left.t_{(15)}=2.62, p=0.02\right)$. No-go trial performance following a correct response inhibition $(M=49 \%, \mathrm{SD}=18 \%)$, which also presented a different stimulus to the No-go trial that preceded it, was also significantly poorer than posterror "same" trial performance $\left(t_{(15)}=3.81, p=0.01\right)$. Postcorrect and posterror "different" No-go trial performance were not significantly different $\left(t_{(15)}=-0.77, p=0.45\right)$.

Correction- and error-predicting errors were further examined to establish other behavioral measures associated with adaptive behavior change. Correcting an error was not associated with a greater interval (number of Go trials) between consecutive No-go trials, with correction-predicting errors $(M=12.9$ Go trials, $\mathrm{SD}=3.9$ ) having a similar interval to error-predicting errors $(M=12.1$ Go trials, $\mathrm{SD}=3.76)$. Similarly, correction- and error-predicting errors had intervals similar to the preceding No-go trial $(M=12.1$ trials, $\mathrm{SD}=5.3$ and $M=11.9$ trials, $\mathrm{SD}=$ 5.1 , respectively), indicating equivalent levels of response prepotency before an error.

Performance on the preceding No-go trial also appeared to influence error correction rates. Task conditions dictated that first-presentation errors could be preceded by an unlimited number of previous correct No-go responses (the data indicate that the range across participants was 1-12 consecutive correct No-go responses), but had to be preceded by at least two consecutive errors (every second No-go error was not predictive of the following No-go stimulus letter). First-presentation errors preceded by two consecutive No-go errors had a $52 \%(\mathrm{SD}=11 \%)$ correction rate, whereas those preceded by two consecutive correct No-go responses had a $76 \%(S D=9 \%)$ correction rate. Taking into account that trial numbers decreased as a function of consecutive correct responses, error correction rate increased as a function of the number of preceding consecutive correct No-go responses, from $69 \%(\mathrm{SD}=12 \%)$ for one preceding correct response, to $70 \%(\mathrm{SD}=11 \%)$ for three, $79 \%$ for four $(\mathrm{SD}=17)$, and $100 \%$ correction $(\mathrm{SD}=11 \%)$ for any error preceded by five or more consecutive correct No-go responses.
Response speed for correctionpredicting errors $(M=300 \mathrm{~ms}, \mathrm{SD}=38.6)$ was not significantly different from response speed for error-predicting errors $\left(M=286 \mathrm{~ms}, \mathrm{SD}=50.5 ; t_{(15)}=1.58, p=\right.$ 0.13 ), although both types of errors were made significantly faster than mean Go trial reaction time (RT) $(M=323 \mathrm{~ms}, \mathrm{SD}$ $=43.4 ; p=0.038$ and 0.009 , respectively). The same pattern of results was seen for repeat errors (i.e., the second error in a pair of consecutive No-go errors) (Fig. 3).

Neither correction or error-predicting errors showed posterror slowing until at least five trials after the No-go error (Fig. 3 ), a phenomenon we have previously seen with Go/No-go tasks (Hester et al., 2007). A 2 (condition) $\times 16$ (trial) repeatedmeasures ANOVA was used to compare posterror slowing across error types using posterror change (a subtraction of posterror Go-trial RT from the preerror Go-trial RT). The analysis revealed a main effect for trial number $\left(F_{(1,15)}=9.27, p=0.001\right)$, with reaction time slowing as a function of interval from the error. There was no main effect of error type, with correction- and error-predicting errors showing similar posterror slowing $\left(17 \mathrm{vs} 19 \mathrm{~ms}\right.$, respectively; $F_{(1,15)}=0.02$, $p=0.88)$. The interaction between error type and trial was also nonsignificant $\left(F_{(1,15)}=1.14, p=0.32\right)$. Posterror slowing occurred more rapidly after repeat errors. The first trial following an error showed posterror speeding (as was seen for first presentation errors), but significant posterror slowing was evident from the second posterror trial onwards.

The Go trial immediately preceding a No-go trial (pre-Nogo), which shared the same stimulus letter with the previous No-go error, was expected to act as a cue to participants by alerting them to the upcoming No-go trial. The pre-No-go Go trial RT before a stop "same" ( $M=360 \mathrm{~ms}, \mathrm{SD}=72.9)$ was significantly slower than that for a repeat error $\left(M=291 \mathrm{~ms}, \mathrm{SD}=52.5 ; t_{(15)}\right.$ $=4.54, p=0.01)$. This effect reflected a general trend, seen previously with the Go/No-go task, wherein the pre-No-go Go trial RT is typically slower before stops than in preerror trials. For example, when participants had correctly inhibited on a No-go trial, the next No-go trial would present a different stimulus, and therefore be unpredictable. Despite this, Go trial RT before a stop $(M=404 \mathrm{~ms}, \mathrm{SD}=119.3)$ was significantly slower than before an error $\left(M=307 \mathrm{~ms}, \mathrm{SD}=35.7 ; t_{(15)}=2.94, p=0.01\right)$. Similarly, when the No-go trial appeared unpredictably (e.g., after a repeat error), Go trial RT before a stop $(M=391 \mathrm{~ms}, \mathrm{SD}=94.4)$ was significantly slower than before an error $(M=301 \mathrm{~ms}, \mathrm{SD}=49.3$; $\left.t_{(12)}=3.34, p=0.01\right)$.

\section{FMRI results: correction- versus error-predicting errors}

No-go errors were associated with significant activity in the posterior medial frontal cortex (Fig. 4). The center of mass for this cluster of activity was located in the right dorsal anterior cingulate cortex (MNI coordinates: $x=2 ; y=12 ; z=44$ ), which falls within the rostral cingulate zone highlighted by Ridderinkhof and colleagues' review of performance monitoring (Ridderinkhof et al., 2004). Within this functionally defined ROI, correction-predicting errors were associated with significantly higher levels of BOLD activity than error-predicting errors. Activity in several other regions also differentiated correction from error-predicting errors (Table 1, Fig. 4), including bilateral insula 


\section{I}

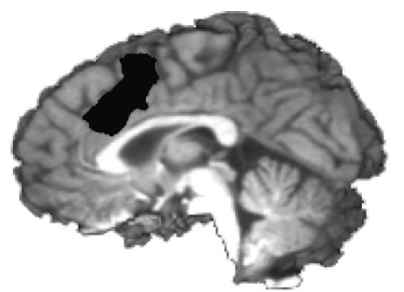

II

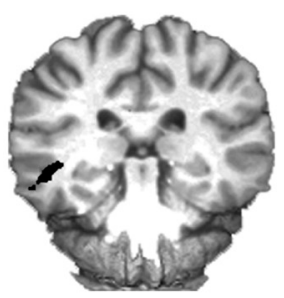

$\mathrm{R}$

L

III

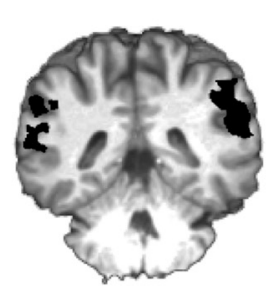

L

$\mathrm{R}$

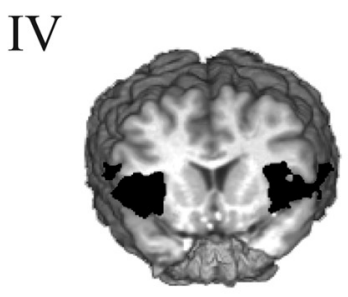

R

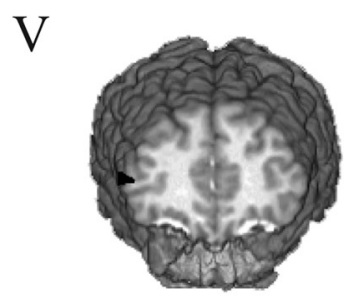

R
L

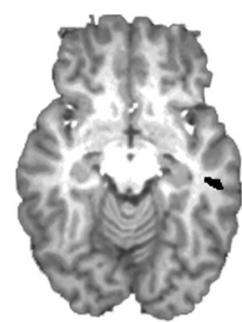

L R
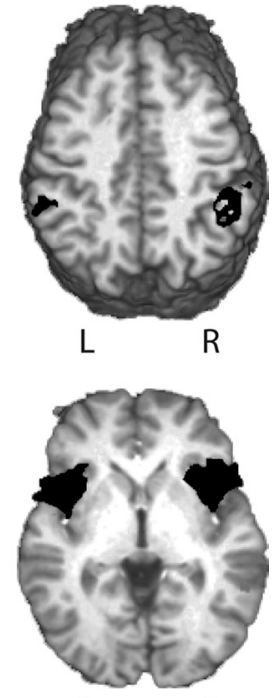

$\mathrm{R}$

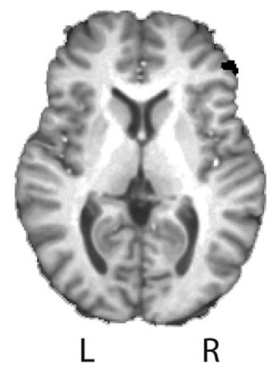

L

L

$\mathrm{R}$

Figure 4. 3D rendering of brain regions differentiating corrected from error-predicting errors. Correction-predicting errors, when compared with error-predicting errors, were associated with significantly higher levels of activity in the following: axial view of the error-related cingulate cluster (MNI: $x=2 ; y=12 ; z=44)(I)$; the right middle temporal gyrus $(x=48 ; y=$ $-27 ; z=-9)(I I)$; the right inferior parietal $(x=53 ; y=-42 ; z=32)$ and left inferior parietal $(x=-54 ; y=-41 ; z=39)$ gyri $(I I I)$; the right insula $(x=40 ; y=14 ; z=5)$ and left insula $(x=-40 ; y=13 ; z=4)(\boldsymbol{I V})$; and the right inferior frontal gyrus $(x=49 ; y=39$; $z=12)(\boldsymbol{V})$.

and bilateral inferior parietal, right middle temporal, and right inferior frontal gyri (pars triangularis). The relationship between activity in these regions was further examined using Pearson correlation coefficients. Participants who had high levels of pMFC activity during correction-predicting errors also had high levels of activity in the right insula $(r=0.78, p=0.01)$, left insula $(r=$

0.87, $p=0.01)$, right middle temporal gyrus (MTG) $(r=0.62$, $p=0.01)$, and right inferior frontal gyrus (IFG) $(r=0.48, p=$ $0.05)$. Significant correlations were also seen between the right insula and several other regions, including the left insula $(r=$ $0.82, p=0.01)$, right middle temporal gyrus $(r=0.53, p=0.03)$, and right inferior frontal gyrus $(r=0.64, p=0.01)$. None of these correlations was evident for error-related activity during errorpredicting errors.

Individual differences in error-related activity also correlated with behavioral performance. Participants who had high levels of activity during correction-predicting errors in the right middle temporal gyrus $(r=0.51, p=0.04)$ and right inferior frontal gyrus $(r=0.49, p=0.05)$ had better error correction rates.

Two error-related regions showed greater activity for errorpredicting errors when compared with correction-predicting errors: the right middle temporal gyrus and the left posterior cingulate gyrus (Table 1). The differences were due to deactivation of these regions during correction-predicting errors. Activity in these two regions was correlated $(r=0.52, p=0.04)$, but did not correlate with activity in the other "correction" regions, nor did activity in these two regions correlate with behavioral performance.

\section{Repeat errors}

Neural activity during repeat adaptive and maladaptive errors was also compared. Significant activity was identified for both adaptive and maladaptive errors in the pMFC region, which did not differ across the two trial types. The comparison of all functionally defined regions revealed two clusters with significantly different activity for the two types of errors: right middle temporal gyrus (MNI coordinates: $x=55 ; y=-50 ; z=4$ ) and thalamus (medial dorsal nucleus) (MNI coordinates: $x=0 ; y=-17$; $z=11$ ). The temporal region showed significant activity during maladaptive repeat errors but no activity during adaptive repeat errors (relative to the Go-trial baseline), and the thalamic region showed significant deactivation during maladaptive errors. None of the regions associated with error correction in the first round showed significant differences in activity that predicted error correction during repeat errors.

\section{Stops}

The event-related BOLD signal during stops indicated significant activity in regions including the right prefrontal, parietal, and anterior cingulate cortices, as previously observed with this task (Hester et al., 2004a,b). Having advance knowledge of the identity of an upcoming No-go trial was predicted to influence BOLD activity during stops, as the pre-No-go Go trial would have acted as a cue to increase cognitive control for the subsequent No-go trial. A comparison of same and different stops revealed significantly greater activity for "same" stops in eight clusters (Table 2), including right inferior frontal, bilateral inferior parietal, right middle temporal, right posterior cingulate, left middle frontal, right precuneus, and left precentral gyri. Bilateral precuneus regions demonstrated deactivation during both types of stops, but the magnitude of deactivation was greater for "same" than for "different" stops.

\section{Discussion}

The results of the present study indicate that adaptive changes to cognitive control behavior, in the form of avoiding consecutive response inhibition errors, are predicted by the magnitude of error-related activity in the pMFC. Behavioral performance measures showed that participants learned from errors; response in- 
Table 2. Regions of stop-related BOLD activity differentiating correct response inhibition for repeated (same stops) and random No-go (different stops) stimuli

\begin{tabular}{|c|c|c|c|c|}
\hline \multirow[b]{2}{*}{ Brain region } & \multirow[b]{2}{*}{ Volume ( $\mu$ l) } & \multicolumn{3}{|c|}{ MNI coordinates } \\
\hline & & $x$ & $y$ & $z$ \\
\hline \multicolumn{5}{|c|}{ Same stops $>$ different stops } \\
\hline R inferior frontal & 17,863 & 27 & 15 & 27 \\
\hline R inferior parietal & 13,456 & 46 & -48 & 38 \\
\hline R middle temporal & 2153 & 52 & -27 & -7 \\
\hline L inferior parietal & 1931 & -44 & -51 & 43 \\
\hline $\mathrm{R}$ middle cingulate & 1029 & 0 & -23 & 29 \\
\hline L middle frontal & 517 & -29 & 48 & 28 \\
\hline R precuneus & 473 & 6 & -74 & 39 \\
\hline L precentral & 181 & -32 & -15 & 55 \\
\hline \multicolumn{5}{|c|}{ Different stops $>$ same stops } \\
\hline L precuneus & 401 & -11 & -55 & 10 \\
\hline Rprecuneus & 300 & 7 & -53 & 13 \\
\hline
\end{tabular}

hibition performance for No-go trials following a commission error was significantly better when the same stimulus was presented (68\% accuracy) than when a different stimulus appeared $(53 \%)$. Categorizing commission errors by subsequent No-go trial performance-essentially whether the failure to accurately withhold a prepotent motor response was corrected at the next presentation of the same No-go trial sequence-revealed that the magnitude of error-related pMFC activity predicted future correction.

A higher level of pMFC activity was observed during correction-predicting errors compared with error-predicting errors, despite the interval between an error event and subsequent correction lasting, on average, 12 trials. Error-predicting errors also had a mean interval of 12 trials, discounting the possibility that error correction was aided by a shorter memory delay. Similarly, the diminished pMFC activity during error-predicting errors cannot easily be explained by participants being unaware of these errors, because the magnitude of error-related pMFC activity has previously been shown to be insensitive to the awareness (or unawareness) of errors (Nieuwenhuis et al., 2001; Hester et al., 2005; Klein et al., 2007a).

The elevated level of pMFC activity during correctionpredicting errors was accompanied by similar effects in other regions, including right inferior frontal gyrus, right middle temporal gyrus, bilateral insula, and bilateral inferior parietal gyri. In support of this within-subject effect, participants with higher levels of BOLD activity during correction-predicting errors in either the right IFG, or right middle temporal regions, had higher error correction rates.

While significant error-related activity was observed in the pMFC region during repeat errors, no relationship between the magnitude of activity and error correction was identified. Unlike initial errors, a second consecutive commission error was followed by a different stimulus letter and therefore offered no learning opportunity. Under these conditions, typical of most No-go tasks, error-related neural activity was not predictive of performance on the following No-go trial.

In contrast with previous studies, and expectations from the conflict-monitoring theory (Botvinick et al., 2001; Yeung et al., 2004), we found no relationship between error-related pMFC activity and posterror changes in response speed. The greater BOLD activity for correction-predicting errors, when compared with error-predicting errors, was not associated with posterror slowing. Posterror slowing has been argued to represent an adaptive posterror change in response behavior, reflecting a phasic increase in cognitive control that results from dealing with the previous difficult trial (Holroyd et al., 2005). The absence of posterror slowing may be related to the Go/No-go task paradigm, a phenomenon we have previously observed (Hester et al., 2007). The percentage of responseincongruent trials in the Go/No-go task is typically lower than other cognitive control paradigms, $10 \%$ for the current version versus the typical $25 \%$ for flanker and Stroop tasks. The lower percentage reduces the possibility that another incongruent trial will be presented immediately, or soon after, an error, which in turn reduces the adaptive benefits of posterror slowing.

The association between error-related pMFC activity and subsequent improvements in performance appears consistent with theories positing a role for the pMFC region in dopaminergically mediated reinforcement learning mechanisms (Holroyd and Coles, 2002; Nieuwenhuis et al., 2004; Brown and Braver, 2005; Frank, 2005). For example, that errorrelated pMFC activity reflects a phasic decrease in midbrain dopamine activity (modulated by the basal ganglia) that indicates that an outcome was "worse than expected." This dopaminergic signal contributes to reinforcement learning through stimulation of other regions in the mesencephalic dopamine system. While not assessing dopamine function directly, the current results demonstrate increased activity in the pMFC for all errors (relative to the Go-trial baseline), regardless of subsequent performance. The relative magnitude of activity was associated with future improvements in performance. An increase in activity during correction-predicting errors in the right IFG, right middle temporal gyrus, bilateral inferior parietal, and bilateral insula regions was also associated with performance improvement. Activity in the pMFC during correction-predicting errors correlated with activity in the right MTG, right IFG, and insula-correlations that were not significant during error-predicting errors.

Consistent with the hypothesis that the pMFC plays an indirect role in driving behavior change through its influence on other task-related regions, the present data indicate that individual differences in error correction rates are not predicted by pMFC activity per se, but by activity in regions that correlate with the pMFC, namely the right IFG and right MTG. We have previously demonstrated a similar predictive relationship using an associative learning task (Hester et al., 2008), in which improvements in recall performance were associated with heightened error-related activity in the pMFC and hippocampus (Klein et al., $2007 b$ ). Again, activity in these two regions was highly intercorrelated during correction-predicting errors, but not during errorpredicting errors. While these data do not demonstrate causal relationships between activity in these regions, it is, however, encouraging for this hypothesis that error-related pMFC activity is associated with predictive activity in cortical regions generally considered critical to the cognitive domain being tested. For example, previous studies have shown a relationship between hippocampal activity and associative learning (Small et al., 2001; Sperling et al., 2001; Degonda et al., 2005; Suzuki, 2007), and right IFG activity and inhibitory control (Konishi et al., 1998; Aron et al., 2004; Chambers et al., 2006).

The right inferior frontal cortex (IFC) has been consistently linked to response inhibition (Aron et al., 2004; Chambers et al., 
2006; Garavan et al., 2006), as well as to the maintenance of working memory (Courtney, 2004). IFC activity in the present contrast would appear to be consistent with a role for this region in working memory: updating working memory with the errorrelated letter stimulus information that would identify the next No-go trial. Similarly, the right middle temporal region associated with improved performance in the current task has also been implicated in the phonological rehearsal of information held in working memory (Buchsbaum and D'Esposito, 2008) and rule learning (Bunge, 2004). Right IFC activity during correct response inhibition has been argued to represent the suppression of an irrelevant response (Aron et al., 2004). While it is not possible directly to ascribe this role here (because activity occurs during a failed response inhibition), previous data suggest that cueing the requirement for response inhibition will activate the right IFC in a preparatory manner (Hester et al., 2004b; Polli et al., 2005).

Current theories argue that different task or stimulus conditions, such as response conflict, error likelihood, or outcome expectancy, influence the magnitude of pMFC activity during an error (Gehring and Willoughby, 2002; Holroyd and Coles, 2002; Nieuwenhuis et al., 2004; Brown and Braver, 2005; Frank, 2005). The present data demonstrate a within-subject relationship between error-related pMFC activity and learning from inhibition errors, but our task was not explicitly designed to test these competing theories because of the requirement to adjust the task parameters to an individual participant's performance. Despite this limitation, two results appear particularly relevant. First, the average number of Go trials preceding corrected and errorpredicting errors and No-go stimulus content (letter identity) was equivalent. The frequency and recency of a response influences the level of competition between responses (Yeung et al., 2004; Holroyd et al., 2005), and in turn the level of response conflict. If correction-predicting errors were associated with higher levels of response conflict, accounting for their higher level of pMFC activity, it might have been predicted that greater response prepotency had accumulated before these No-go trials. Consequently, the present data offer no obvious evidence that correction- and error-predicting errors differed in their levels of response competition.

Second, a correction-predicting error was significantly more likely if it had been preceded by successful No-go performance, rather than an error. The likelihood of error correction appeared to increase linearly as a function of the preceding number of consecutive stops, from $69 \%$ correction when preceded by one stop, up to $100 \%$ correction for errors preceded by five or more stops. This pattern of results appears consistent with theories that suggest that error-related pMFC activity is influenced by outcome expectancy, with higher levels of activity for outcomes that were worse than expected (Gehring and Willoughby, 2002; Holroyd and Coles, 2002; Nieuwenhuis et al., 2004; Frank, 2005), or occurred during periods when error likelihood was lower (Brown and Braver, 2005). These models of error-related neural activity argue that the magnitude of activity in the pMFC region reflects the extent to which the actual outcome is better or worse that the predicted outcome. Predictions are based on recent experience, so that during a period of continued success the expectation of a successful outcome increases.

To conclude, the current findings provide new evidence for an association between error-related pMFC activity and adaptive posterror behavior change. By examining response inhibition using a task that provides an opportunity to learn from errors, we have revealed a neural correlate for the hypothesized association between error-related activity and subsequent adaptive behavior change. The results provide the first evidence that cognitive control performance can be predicted from error-related activity occurring up to 20 trials (or seconds) earlier. The increased likelihood of adaptive change when participants had recently been successful is consistent with models arguing for the influence of outcome expectancy on error-related activity. Demonstrating this brain-behavior relationship in healthy controls may help to explain the consistent finding of diminished error-related activity and increased propensity for perseverative behavior in a diverse range of clinical conditions, including schizophrenia and drug dependence.

\section{References}

Aron AR, Robbins TW, Poldrack RA (2004) Inhibition and the right inferior frontal cortex. Trends Cogn Sci 8:170-177.

Botvinick MM, Braver TS, Barch DM, Carter CS, Cohen JD (2001) Conflict monitoring and cognitive control. Psychol Rev 108:624-652.

Brown JW, Braver TS (2005) Learned predictions of error likelihood in the anterior cingulate cortex. Science 307:1118-1121.

Buchsbaum BR, D'Esposito M (2008) The search for the phonological store: from loop to convolution. J Cogn Neurosci 20:762-778.

Bunge SA (2004) How we use rules to select actions: a review of evidence from cognitive neuroscience. Cogn Affect Behav Neurosci 4:564-579.

Chambers CD, Bellgrove MA, Stokes MG, Henderson TR, Garavan H, Robertson IH, Morris AP, Mattingley JB (2006) Executive "brake failure" following deactivation of human frontal lobe. J Cogn Neurosci 18:444-455.

Courtney SM (2004) Attention and cognitive control as emergent properties of information representation in working memory. Cogn Affect Behav Neurosci 4:501-516.

Cox RW (1996) AFNI: software for analysis and visualization of functional magnetic resonance neuroimages. Comput Biomed Res 29:162-173.

Debener S, Ullsperger M, Siegel M, Fiehler K, von Cramon DY, Engel AK (2005) Trial-by-trial coupling of concurrent electroencephalogram and functional magnetic resonance imaging identifies the dynamics of performance monitoring. J Neurosci 25:11730-11737.

Degonda N, Mondadori CR, Bosshardt S, Schmidt CF, Boesiger P, Nitsch RM, Hock C, Henke K (2005) Implicit associative learning engages the hippocampus and interacts with explicit associative learning. Neuron 46:505-520.

Falkenstein M, Hohnsbein J, Hoormann J, Blanke L (1991) Effects of crossmodal divided attention on late ERP components. II. Error processing in choice reaction tasks. Electroencephalogr Clin Neurophysiol 78:447-455.

Frank MJ (2005) Dynamic dopamine modulation in the basal ganglia: a neurocomputational account of cognitive deficits in medicated and nonmedicated Parkinsonism. J Cogn Neurosci 17:51-72.

Garavan H, Ross TJ, Stein EA (1999) Right hemispheric dominance of inhibitory control: an event-related functional MRI study. Proc Natl Acad Sci U S A 96:8301-8306.

Garavan H, Ross TJ, Murphy K, Roche RA, Stein EA (2002) Dissociable executive functions in the dynamic control of behavior: inhibition, error detection, and correction. Neuroimage 17:1820-1829.

Garavan H, Hester R, Murphy K, Fassbender C, Kelly C (2006) Individual differences in the functional neuroanatomy of inhibitory control. Brain Res 1105:130-142.

Gehring WJ, Willoughby AR (2002) The medial frontal cortex and the rapid processing of monetary gains and losses. Science 295:2279-2282.

Gehring WJ, Goss B, Coles MGH, Meyer DE, Donchin E (1993) A neural system for error detection and compensation. Psychol Sci 4:385-390.

Hebb DO (1961) Brain mechanisms and learning. In: Distinctive features of learning in the higher animal (Delafresnaye JF, ed). New York: Oxford UP.

Hester R, Murphy K, Garavan H (2004a) Beyond common resources: the cortical basis for resolving task interference. Neuroimage 23:202-212.

Hester RL, Murphy K, Foxe JJ, Foxe DM, Javitt DC, Garavan H (2004b) Predicting success: patterns of cortical activation and deactivation prior to response inhibition. J Cogn Neurosci 16:776-785.

Hester R, Foxe JJ, Molholm S, Shpaner M, Garavan H (2005) Neural mechanisms involved in error processing: a comparison of errors made with and without awareness. Neuroimage 27:602-608.

Hester R, Barre N, Mattingley JB, Foxe JJ, Garavan H (2007) Avoiding an- 
other mistake: error and posterror neural activity associated with adaptive posterror behavior change. Cogn Affect Behav Neurosci 7:317-326.

Hester R, Barre N, Murphy K, Silk TJ, Mattingley JB (2008) Human medial frontal cortex activity predicts learning from errors. Cereb Cortex 18:1933-1940.

Holroyd CB, Coles MG (2002) The neural basis of human error processing: reinforcement learning, dopamine, and the error-related negativity. Psychol Rev 109:679-709.

Holroyd CB, Yeung N, Coles MG, Cohen JD (2005) A mechanism for error detection in speeded response time tasks. J Exp Psychol Gen 134:163-191.

Keppel G (1991) Design and analysis: a researcher's handbook. Englewood Cliffs, NJ: Prentice Hall.

Kerns JG, Cohen JD, MacDonald AW 3rd, Cho RY, Stenger VA, Carter CS (2004) Anterior cingulate conflict monitoring and adjustments in control. Science 303:1023-1026.

Klein TA, Endrass T, Kathmann N, Neumann J, von Cramon DY, Ullsperger M (2007a) Neural correlates of error awareness. Neuroimage 34:1774-1781.

Klein TA, Neumann J, Reuter M, Hennig J, von Cramon DY, Ullsperger M (2007b) Genetically determined differences in learning from errors. Science 318:1642-1645.

Konishi S, Nakajima K, Uchida I, Sekihara K, Miyashita Y (1998) No-go dominant brain activity in human inferior prefrontal cortex revealed by functional magnetic resonance imaging. Eur J Neurosci 10:1209-1213.

Murphy K, Garavan H (2005) Deriving the optimal number of events for an event-related fMRI study based on the spatial extent of activation. Neuroimage 27:771-777.
Nieuwenhuis S, Ridderinkhof KR, Blom J, Band GP, Kok A (2001) Errorrelated brain potentials are differentially related to awareness of response errors: evidence from an antisaccade task. Psychophysiology 38:752-760.

Nieuwenhuis S, Holroyd CB, Mol N, Coles MG (2004) Reinforcementrelated brain potentials from medial frontal cortex: origins and functional significance. Neurosci Biobehav Rev 28:441-448.

Polli FE, Barton JJ, Cain MS, Thakkar KN, Rauch SL, Manoach DS (2005) Rostral and dorsal anterior cingulate cortex make dissociable contributions during antisaccade error commission. Proc Natl Acad Sci U S A 102:15700-15705.

Ridderinkhof KR, Ullsperger M, Crone EA, Nieuwenhuis S (2004) The role of the medial frontal cortex in cognitive control. Science 306:443-447.

Small SA, Nava AS, Perera GM, DeLaPaz R, Mayeux R, Stern Y (2001) Circuit mechanisms underlying memory encoding and retrieval in the long axis of the hippocampal formation. Nat Neurosci 4:442-449.

Sperling RA, Bates JF, Cocchiarella AJ, Schacter DL, Rosen BR, Albert MS (2001) Encoding novel face-name associations: a functional MRI study. Hum Brain Mapp 14:129-139.

Suzuki WA (2007) Making new memories: the role of the hippocampus in new associative learning. Ann N Y Acad Sci 1097:1-11.

Ullsperger M, von Cramon DY (2003) Error monitoring using external feedback: specific roles of the habenular complex, the reward system, and the cingulate motor area revealed by functional magnetic resonance imaging. J Neurosci 23:4308-4314.

Yeung N, Botvinick MM, Cohen JD (2004) The neural basis of error detection: conflict monitoring and the error-related negativity. Psychol Rev 111:931-959. 